\title{
Hypertensive target organ damage and its relationship with platelet indices
}

\author{
Siva Ranganathan Green*, Lavanya Vetrivel
}

Department of General Medicine, Mahatma Gandhi Medical College and Research Institute, Pondicherry, India

Received: 28 April 2021

Revised: 18 June 2021

Accepted: 19 June 2021

\section{*Correspondence:}

Siva Ranganathan Green,

E-mail: dr.siva.green@gmail.com

Copyright: (c) the author(s), publisher and licensee Medip Academy. This is an open-access article distributed under the terms of the Creative Commons Attribution Non-Commercial License, which permits unrestricted non-commercial use, distribution, and reproduction in any medium, provided the original work is properly cited.

\begin{abstract}
Hypertension affects vascular endothelium of retina, kidney, and heart. These are called as target organ damage (TOD). Hypertension causes endothelial damage by shear mechanical stress which leads to platelet aggregation and activation. This article is a review for prediction of TOD in hypertensives by cost effective routine indicators like platelet indices [platelet distribution width (PDW), mean platelet volume (MPV) and platelet count]. Hypertension mediated target organ damage like left ventricular hypertrophy, hypertensive retinopathy and nephropathy is associated with increased platelet indices like MPV, PDW, and PLT. The use of these cost-effective platelet indicators in newly diagnosed hypertensives may need further studies to have clinical implications.
\end{abstract}

Keywords: Hypertension, Organ damage, Platelet indices

\section{INTRODUCTION}

Hypertension is one of the most common modifiable risk factors for cardiovascular events. ${ }^{1}$ Hypertension being asymptomatic especially during initial days, increases the risk of target organ damage (TOD) like renal failure, retinal damage and myocardial infarction. ${ }^{2}$

Exposure of circulating platelets to the mechanical shear forces in the hypertensives results in specific cellular interactions that are responsible for the increased platelet activation which are reflected in platelet indices like mean platelet volume (MPV), platelet distribution width (PDW) and platelet count (PLT). ${ }^{3}$

An electronic internet search was used to select the English literature from 2000 to until 2020 using databases like PubMed, Google Scholar and ProQuest with hypertension, target organ damage and platelet indices as keywords. Totally 31 papers were selected excluding studies done on children and pregnant participants.

\section{DEFINITION OF HYPERTENSION}

(a) The 2020 European guidelines define hypertension as office blood pressure $\geq 140 \mathrm{mmHg}$ and diastolic $\geq 90$ mmHg. ${ }^{4}$ The diagnosis should not be made on a single office visit. Usually, 2-3 office visits are required to confirm the diagnosis. If the blood pressure is $\geq 180 / 110$ $\mathrm{mmHg}$, and if there is evidence of cardiovascular disease, hypertension can be diagnosed in a single visit. ${ }^{1}$ (b) The 2019 American guidelines lower the threshold for hypertension based on a large meta-analysis SPRINT trial with systolic blood pressure $\geq 130 \mathrm{mmHg}$ and diastolic BP $\geq 80 \mathrm{mmHg}$ to define hypertension. ${ }^{5}$

\section{TARGET ORGAN DAMAGE AND PLATELET INDICES}

Hypertension mediated TOD is defined as a structural or functional alteration in arterial vasculature and the organ it supply. ${ }^{1}$ The most commonly involved target organs include the heart, kidney, retina, central and peripheral 
arteries. ${ }^{1}$ Assessment of cardiovascular (CV) risk plays an important role in the management of hypertension; TOD will provide important therapeutic guidance on the management of hypertensive patients with low or moderate $\mathrm{CV}$ risk. It will also help in the preferential selection of drug treatment based on the specific impact on TOD. ${ }^{1}$ The effect of high blood pressure on the vital organs may be mediated by activation of the coagulation system via endothial injury, and this might have effects on platelets.

Platelet activation plays a critical role in the pathophysiology of thrombotic events and hypertensionrelated target organ damages. ${ }^{6}$ In order to assess cardiovascular risk and to clarify the pathogenesis of atherothrombosis, some biochemical markers are used. One of them is the platelet indices (MPV, PDW and PLT), a potential marker of platelet activity. ${ }^{7}$ The effect of an over-activated sympathetic nervous system on the hemostatic system occurs in two ways; first, the platelet activation via alpha two adrenoreceptors stimulation, which causes a change in shape, thereby increases MPV. Second, larger the activated platelets, which are sequestered in the spleen, can be released into the circulatory system following the elevated levels of adrenaline that contribute to increased PDW; this might be the first mechanism for increased levels of MPV. ${ }^{7}$ PLT appear to be independently associated with TOD in patients with HTN. High PDW values may indicate higher production of larger reticulated platelets. Thus, changes in platelets reflected by PDW and platelet count may reveal hypertension status and predict thrombotic risk and TOD.

\section{Hypertensive heart disease and platelet indices}

Hypertensive heart disease is usually asymptomatic and manifests clinically only in advance stages as angina pectoris, systolic and diastolic heart failure, and arrhythmias. $^{8}$ These patients have increase in left ventricular mass. Symptoms in hypertensive heart disease are due to structural and functional changes in the left ventricle, left atrium, and coronary arteries secondary to chronically elevated blood pressure. ${ }^{2}$ Chronically elevated blood pressure will cause endothelial dysfunction, which predisposes the patients to develop coronary artery diseases and peripheral arterial disease and places the patient at higher risk for atherosclerotic disease. ${ }^{3}$

Active platelets contain greater MPV, PDW values and platelet count, and the coagulation of active platelets or local thrombosis would further increase the abnormality of PDW and platelet count. ${ }^{9}$ Platelet indices, especially MPV, have prognostic indications with cardiovascular settings; their prognostic value is mainly linked to oxidative stress and inflammation. Large platelets are more active metabolically and enzymatically as they contain more dense granules, and they have increased thrombotic potential compared with small platelets. $^{9}$ Increasing platelet indices are associated with left ventricular hypertrophy as activated platelets dissipate fatty acids and activation of RAAS, causing cardiomyocyte growth and interstitial fibrosis, which are hallmarks of $\mathrm{LVH}$ in hypertensive individuals. ${ }^{10}$

\section{Hypertensive mediated kidney disease and platelet indices}

Hypertension-mediated kidney damage expresses the diffuse involvement of renal blood vessels. The HOT study showed even a mild increase in serum creatine (1.5 $\mathrm{mg} / \mathrm{dl}$ ) would increase the risk of cardiovascular death by three times. ${ }^{11}$ The most common clinical presentation of hypertensive kidney disease is an asymptomatic rise in serum creatinine, which denote renal parenchymal damage. ${ }^{12}$ Albuminuria has been regarded as a sign of hypertensive renal damage. ${ }^{13}$

A mild increase in urine albumin excretion is called microalbuminuria. It is a powerful predictor of cardiovascular mortality and is an early predictor of endothelial dysfunction in hypertensive patients. ${ }^{14}$ Increased platelet indices (MPV, PDW, PCT) which leads to increased production of tromboxinase which causes smooth muscle activation and proliferation which predisposes to development of microalbuminuria. ${ }^{10}$ The ASCOT trial demonstrated that hypertensive patients with target organ damage, including stroke, previous MI, angina, microalbuminuria and left ventricular hypertrophy, had higher MPV levels than hypertensive patients without TOD. ${ }^{10}$ Guagnano et al and Ferroni et al found a positive linear association between platelet activation and presence of microalbuminuria in their study this is attributed to high blood pressure and the endothelial dysfunction, which causes activation of platelets this will manifest as increased MPV and PDW. ${ }^{15}$ Endothelial dysfunction in hypertensives with microalbuminuria are reflected by decreased levels of $\mathrm{NO}$ and increased plasma levels of ICAM-1 in the patients with microalbuminuria. ${ }^{15}$ Cause of microalbuminuria can be traced to structural and functional transformational process in the glomeruli (endothelium, glomerular basal membrane, podocytes) that are associated with increased permeability. Interestingly, this permeability disorder is not limited to the renal vessels but can be observed in the entire vascular system. Microalbuminuria is the renal manifestation of generalized endothelial dysfunction. ${ }^{16}$

\section{Hypertensive retinopathy and platelet indices}

Hypertension affects the eye causes choroidopathy, retinopathy and optic neuropathy. ${ }^{17}$ Retinal blood vessels have characteristic features such as absence of sympathetic nerve supply and presence of blood-retinal barrier. ${ }^{17}$ Coban et al found a positive relation between MPV and hypertensive retinopathy in correlation analysis. The activated platelets can aggravate the process of hypertension mediated retinal damage causing higher grades of hypertensive retinopathy. ${ }^{18}$ Hypertension by means of endothelial dysfunction causes platelet activation. The activated platelets can aggravate the process of hypertension mediated retinal damage causing higher grades of hypertensive retinopathy. ${ }^{18}$ 
Table 1: Comparison of variables between groups A and $\mathbf{B}$.

\begin{tabular}{|llll|}
\hline Variables & Group A & Group B & Significance \\
\hline Platelet- PDW & $14.98 \pm 0.75$ & $15.88 \pm 0.95$ & $<0.001$ \\
\hline Platelet- MPV (fL) & $8.44 \pm 0.23$ & $9.19 \pm 0.47$ & $<0.001$ \\
\hline Platelet- Count & $3.58 \pm 0.16$ & $3.83 \pm 0.17$ & $<0.001$ \\
\hline LV hypertrophy- ECG platelet- PDW & $16.45 \pm 0.42$ & $15.07 \pm 0.85$ & $<0.001$ \\
\hline LV hypertrophy- ECG platelet- MPV (fL) & $9.49 \pm 0.34$ & $8.62 \pm 0.34$ & $<0.001$ \\
\hline LV hypertrophy- ECG platelet- count & $3.93 \pm 0.09$ & $3.64 \pm 0.17$ & $<0.001$ \\
\hline LV hypertrophy- ECHO platelet- PDW & $16.01 \pm 0.83$ & $15.01 \pm 0.87$ & $<0.001$ \\
\hline LV hypertrophy- ECHO platelet- MPV (fL) & $9.26 \pm 0.44$ & $8.52 \pm 0.33$ & $<0.001$ \\
\hline LV hypertrophy- ECHO platelet- count & $3.85 \pm 0.16$ & $3.60 \pm 0.15$ & $<0.001$ \\
\hline Microalbuminuria platelet- PDW & $16.09 \pm 0.77$ & $15.16 \pm 0.94$ & $<0.001$ \\
\hline Microalbuminuria platelet- MPV (fL) & $9.33 \pm 0.44$ & $8.62 \pm 0.38$ & $<0.001$ \\
\hline Microalbuminuria platelet- count & $3.86 \pm 0.14$ & $3.66 \pm 0.20$ & $<0.001$ \\
\hline Retinopathy (KW)- left platelet- PDW & $16.18 \pm 0.73$ & $15.00 \pm 0.76$ & $<0.001$ \\
\hline Retinopathy (KW)- left platelet- MPV (fL) & $9.37 \pm 0.45$ & $8.47 \pm 0.24$ & $<0.001$ \\
\hline Retinopathy $(\mathbf{K W})$ - left platelet- count & $3.87 \pm 0.14$ & $3.58 \pm 0.15$ & $<0.001$ \\
\hline Retinopathy (KW)- right platelet- PDW & $16.18 \pm 0.73$ & $15.00 \pm 0.76$ & $<0.001$ \\
\hline Retinopathy (KW)- right platelet- MPV (fL) & $9.37 \pm 0.45$ & $8.47 \pm 0.24$ & $<0.001$ \\
\hline Retinopathy (KW)- right platelet- count & $3.87 \pm 0.14$ & $3.58 \pm 0.15$ & $<0.001$ \\
\hline
\end{tabular}

Note: Group A- patients without TOD; and Group B- patients with TOD.

\section{CONCLUSION}

Hypertension mediated target organ damage like left ventricular hypertrophy, hypertensive retinopathy and nephropathy is associated with increased platelet indices like MPV, PDW, and PLT. The use of these cost-effective platelet indicators in newly diagnosed hypertensives may need further studies to have clinical implications.

Funding: No funding sources Conflict of interest: None declared

Ethical approval: Not required

\section{REFERENCES}

1. Unger T, Borghi C, Charchar F, Khan NA, Poulter NR, Prabhakaran D, et al. 2020 International Society of Hypertension Global Hypertension Practice Guidelines. Hypertension. 2020;75(6):1334-57.

2. Saju MD, Allagh KP, Scaria L, Joseph S, Thiyagarajan JA. Prevalence, Awareness, Treatment, and Control of Hypertension and Its Associated Risk Factors: Results from Baseline Survey of SWADES Family Cohort Study. Int J Hypertens. 2020;4964835.

3. Tackling G, Borhade MB. Hypertensive Heart Disease. StatPearls. 2020.

4. Williams B, Mancia G, Spiering W, Agabiti RE, Azizi M, Burnier M, et al. 2018 ESC/ESH Guidelines for the management of arterial hypertension. Eur Heart J. 2018;39(33):3021-104.

5. Chopra HK, Ram CVS. Recent Guidelines for Hypertension. Circ Res. 2019;124(7):984-6.
6. Madavi T, Bhole P, Holay MP. Mean platelet volume as a predictor of clinical outcomes in patients of acute myocardial infarction. Int $\mathrm{J}$ Res Med Sci. 2019;7(2):388-93.

7. Lande K, Gjesdal K, Fonstelien E, Kjeldsen SE, Eide I. Effects of adrenaline infusion on platelet number, volume and release reaction. Thromb Haemost. 1985;54(2):450-3.

8. Schmieder RE. End organ damage in hypertension. Dtsch Arztebl Int. 2010;107(49):866-73.

9. Yarlioglues M, Kaya MG, Ardic I, Dogdu O, Kasapkara HA, Gunturk E, et al. Relationship between mean platelet volume levels and subclinical target organ damage in newly diagnosed hypertensive patients. Blood Press. 2011;20(2):92-7.

10. Nadar SK, Blann AD, Kamath S, Beevers DG, Lip GYH. Platelet indexes in relation to target organ damage in high-risk hypertensive patients. J Am Coll Cardiol. 2004;44(2):415-22.

11. Piskorz D. Hypertensive Mediated Organ Damage and Hypertension Management. How to Assess Beneficial Effects of Antihypertensive Treatments?. High Blood Press Cardiovasc Prev. 2020;27(1):9-17.

12. Bicescu G. Epidemiology of Hypertensive Kidney Disease: Diagnosis. Mædica. 2010;5(4):309-10.

13. Viazzi F, Pontremoli R. Blood pressure, albuminuria and renal dysfunction: the 'chicken or egg' dilemma. Nephrol Dial Transplant. 2014;29(8):1453-5.

14. Bidani AK, Griffin KA. Pathophysiology of Hypertensive Renal Damage. Hypertension. 2004;44(5):595-601.

15. Gbadegesin A, Okunola O, Ayodele O, Arogundade F, Sanusi A, Akinsola A. Renal risk profiling in newly diagnosed hypertensives in an urban 
population in Nigeria. Afr Health Sci. 2019;19(4):2863-73.

16. Palatini P. Microalbuminuria in hypertension. Curr Hypertens Rep. 2003;5(3):208-14.

17. Modi P, Arsiwalla T. Hypertensive Retinopathy. StatPearls. StatPearls. 2020.
18. Chen X, Meng Y, Li J, She H, Zhao L, Zhang J, et al. Serum uric acid concentration is associated with hypertensive retinopathy in hypertensive chinese adults. BMC Ophthalmol. 2017;17:83.

Cite this article as: Green SR, Vetrivel L.

Hypertensive target organ damage and its relationship with platelet indices. Int J Adv Med 2021;8:1002-5. 